\title{
Pour une approche explicative et une pratique raisonnée de l'anglais
}

\section{Philippe Rapatel}

\section{OpenEdition}

\section{Journals}

Édition électronique

URL : http://journals.openedition.org/asp/3552

DOI : 10.4000/asp.3552

ISSN : 2108-6354

\section{Éditeur}

Groupe d'étude et de recherche en anglais de spécialité

\section{Édition imprimée}

Date de publication : 1 décembre 1996

Pagination : 253-264

ISSN : 1246-8185

\section{Référence électronique}

Philippe Rapatel, « Pour une approche explicative et une pratique raisonnée de l'anglais », ASp [En ligne], 11-14 | 1996, mis en ligne le 09 mai 2013, consulté le 19 avril 2019. URL : http:// journals.openedition.org/asp/3552 ; DOI : 10.4000/asp.3552

Ce document a été généré automatiquement le 19 avril 2019

Tous droits réservés 


\title{
Pour une approche explicative et une pratique raisonnée de l'anglais
}

\author{
Philippe Rapatel
}

$1 \quad$ Face aux difficultés des étudiants spécialistes d'autres disciplines - que nous baptiserons SPAD pour plus de facilité - qui ne cessent de présenter la grammaire comme leur problème majeur rencontré en langue anglaise, nous avons voulu approfondir la question et, à l'aide d'un questionnaire, leur faire préciser ce qui était pour eux un point délicat, voire douloureux - la source de tous leurs maux. Il est ressorti de cette enquête que, par grammaire, il faut entendre essentiellement le groupe verbal et, au sein de celui-ci, les temps grammaticaux qui, eux-mêmes, recouvraient également aspects et modaux. Ces résultats se sont trouvés confirmés au cours d'une enquête similaire menée auprès d'étudiants anglicistes. Ce premier bilan suscite deux remarques. D'une part il est à noter une fois de plus que les difficultés réelles de l'oral sont peu souvent évoquées en dépit de la pauvre qualité de performance de nos étudiants dans ce domaine, alors qu'il y a beaucoup à surmonter et maîtriser en la matière: accentuation et intonation, notamment, qui font très vite la différence entre un anglophone et un étudiant angliciste. D'autre part le caractère prescriptif, voire normatif, de la grammaire qu'ils ont étudiée jusqu'à présent fait de celle-ci un savoir figé et volumineux, porteur de nombreuses règles qu'ils apparenteraient volontiers à celui d'une langue dite morte.

2 Les étudiants concernés par le nouveau regard que nous voulons leur faire porter sur la langue anglaise, et sa grammaire en particulier, sont des juristes de deuxième année dont l'attente langagière se situe plus au niveau de la communication que de la réflexion linguistique. Rien d'étonnant à cela compte tenu de l'approche essentiellement, souvent uniquement communicative qui leur a été présentée au cours des années précédant leur entrée à l'université et dont ils souhaitent un prolongement efficace dans l'optique de leurs futures carrières où les relations internationales exigent, en effet, une capacité de communication, principalement en anglais. Mais, paradoxalement (ou, logiquement ?) la pauvreté grammaticale du notionnel-fonctionnel nouvelle mouture leur a imposé parallèlement un apprentissage relativement sclérosé de ce qui forme l'ossature de la 
langue. Dans ce contexte, est-il souhaitable de les faire réfléchir sur la langue au risque d'altérer le bel enthousiasme et la spontanéité engendrés par la démarche communicative? C'est l'hypothèse que nous formulons, et l'expérience que nous avons voulu tenter avec nos étudiants juristes.

3 Afin de mesurer l'éventuel bénéfice ou, au contraire, les dégâts de notre approche, nous avons travaillé avec deux classes d'une quarantaine d'étudiants chacune pendant deux années universitaires consécutives, l'une constituant le groupe-test et l'autre le groupetémoin. Le premier groupe travaillerait dans une optique explicative de la langue anglaise, fondée sur une base énonciative, et l'autre recevrait un enseignement traditionnel, uniquement descriptif.

\section{Les pré-tests}

Il s'agit avant tout de procéder à une évaluation des pré-requis, qui permette à la fois de faire un état des lieux grâce auquel les deux groupes pourront être comparés au départ, et de mettre en place des types de tests qui se retrouveront en évaluation sommative en fin d'année afin de mesurer le chemin parcouru par chacun des groupes.

5 Nécessité sera de détailler la qualité évaluative des tests présentés, ce que nous nous proposons de faire lors d'un prochain article. Nous pouvons cependant déjà préciser qu'il s'agit d'un test de sélection (QCM), un test de production semi-guidée (test lacunaire) et d'un exercice d'analyse d'énoncés. Le QCM présente 40 items comportant six options chacun : une clé, deux distracteurs, une option fondamentalement erronée, une option zéro signifiant qu'aucune des quatre options précédentes n'est acceptable et, enfin, la possibilité pour le candidat de dire qu'il ne sait pas. À ceci s'ajoutent quatre C (C1, C2, C3, C4) signalant le degré de certitude du candidat et qu'il devra impérativement indiquer (durée de l'épreuve : une heure).

6 L'exercice de production semi-guidée demande aux étudiants d'écrire à la forme appropriée la base verbale présente dans chaque énoncé proposé et de justifier leur choix. L'étude des résultats se fera sur deux fronts : d'une part l'adéquation de la forme choisie au contexte et, d'autre part, la cohérence entre le choix et la justification. Durée : une heure.

7 L'analyse d'énoncés s'effectue à partir d'énoncés isolés (peu contextualisés) pour lesquels les candidats doivent expliquer la présence de telle forme verbale dans leur propre métalangage (durée de l'épreuve : une heure).

En réponse à leur attente, nous avons principalement orienté nos tests sur l'emploi des temps grammaticaux, aspects et modaux.

\section{Analyse des erreurs}

Il n'est pas question ici de s'attarder sur le détail des erreurs dont nous retiendrons simplement les trois grandes familles suivantes : 1) calques (ou transferts) où l'étudiant modèle L2 sur L1: *I am here since $\left.10 o^{\prime} c l o c k, 2\right)$ grammaire intériorisée erronée par laquelle l'étudiant se construit ses propres règles, généralement dues à la mauvaise intégration des règles de la grammaire prescriptive : « toujours le présent après when », 3) correction de la phrase mais inadéquation au contexte : I've been here for an hour pour I am here for an hour (ou vice versa). 
10 On s'aperçoit, d'une manière générale, que ces erreurs sont d'abord dues au manque de prise en compte du contexte, puis à une grammaire intériorisée défaillante et, en dernier recours lorsque l'étudiant se trouve impuissant face à la tâche, au calque psychologiquement rassurant sur la langue française qui, selon lui, ne doit pas être si éloignée de la langue anglaise.

11 À l'exception du calque, la plupart des erreurs sont le fruit d'une grammaire mal intégrée, parce qu'elle a sans doute été mal présentée. Fort nombreux sont encore les ouvrages, grammaires pédagogiques et précis grammaticaux de manuels scolaires, qui ont une approche prescriptive discutable et un regard descriptif restreint sur les faits de langue, et qui se limitent à l'énoncé de règles et exceptions dont le moins qu'on puisse dire est qu'elles ne vont pas dans le sens de la réflexion de l'apprenant.

\section{Les grammaires pédagogiques}

12 Il n'est nullement question de faire ici le procès d'ouvrages qui s'efforcent de présenter les faits de langue de diverses manières et avec plus ou moins de bonheur. Les exemples figurant en annexe (cf. Annexe I) donnent une idée du type d'approximation, d'inexactitude voire d'aberration qu'il nous est donné de lire ici ou là. Une remarque, concernant une tendance fâcheuse dans le domaine de la publication actuelle, s'impose cependant. Il s'agit de la prolifération d'ouvrages présentant les faits de langue dans un classement alphabétique, ce qui s'apparente plus au mode d'emploi qu'à une grammaire digne de ce nom. Procéder à un tel ordonnancement de $\mathrm{A}$ à $\mathrm{Z}$ c'est priver la langue de toute sa cohérence et de sa logique internes au profit d'une facilité d'accès bien contestable. Commencer à $A(n)$ et terminer à Zero est tout aussi incongru que de vouloir faire imaginer à un extra-terrestre ce qu'est l'être humain par un livre s'ouvrant sur Abdomen et se refermant sur Zygomatique. Où est la cohésion? Où est la cohérence ? Où est la langue et où sont ses opérations profondes, ses invariants, ses valeurs centrales ou fondamentales, ses mécanismes partagés avec d'autres langues indo-européennes ? Nous n'insisterons pas davantage sur ce type d'ouvrage, dont on peut estimer qu'ils sont en grande partie responsables des erreurs que nous reprochons à nos étudiants - en fait des élèves appliqués qui ne font que reproduire des schémas erronés et mémorisés en toute confiance.

13 L'honnêteté nous conduit cependant à montrer que derrière la confusion il y a toujours une raison et qu'il nous est facile, fort du recul et de l'enrichissement des derniers travaux linguistiques, de critiquer ce qui n'a pu bénéficier de ces recherches. Nous nous en remettons alors à cette remarque pertinente de Rotgé :

Il est aisé de faire le procès d'une théorie $\mathrm{X}$ à l'aide d'une théorie $\mathrm{Y}$, la dernière en

date reprochant à son aînée de ne pas avoir vu ce qu'elle a si clairement perçu.

(1993 : 49)

La sagesse de cette citation fait office de transition vers ce que l'on pourrait appeler les ouvrages de "dernière génération ", qu'ils soient purement scolaires comme les manuels de second, voire de premiers cycles de lycée et collège, ou grammaires à l'usage des étudiants. Un nombre fort heureusement croissant de ces publications offre à l'apprenant des pistes de réflexion, des exercices ouvrant à une pratique raisonnée de la langue et l'occasion de s'affranchir du carcan des règles et de leur kyrielle d'exceptions dans une optique explicative (cf. Annexe II). Le choix judicieux et la composition inspirée de certains exercices donnent à ces derniers un statut particulier et un rôle prépondérant 
dans l'acquisition des opérations en L2, qui permettent de dépasser le cadre traditionnel de l'application aveugle et éclairent ainsi l'apprenant dans sa démarche d'autosensibilisation, d'auto-apprentissage et d'auto-évaluation, en un mot, d'autonomie.

Il serait certes insensé et malhonnête de rejeter en bloc tout ce qui émane de l'approche descriptive de la grammaire. En effet, des travaux considérables et hautement louables par leur exhaustivité, tels que ceux de Quirk et al. et de Jespersen, constituent une base solide sur laquelle l'enseignant doit pouvoir s'appuyer pour mener à bien sa propre approche explicative, qui ne saurait exister sans les efforts de tels auteurs. À partir de ces ouvrages complets, c'est à l'enseignant de mesurer la limite du système règle-exception, lequel ne peut aboutir qu'à un apprentissage mécanique à des lieues de la réflexion souhaitée. La question est de savoir si des étudiants juristes de deuxième année ont droit à un type de démarche qui ferait d'eux plus des apprenants-linguistes que des communicatifs.

\section{P.R.L. : pratique raisonnée de la langue}

16 Ce sigle et son développement ne cessent d'être employés lors des concours de recrutement (CAPES, Agrégation) tant par les candidats que par le jury. Cette formule magique qui recouvre souvent divers domaines explorés avec plus ou moins de bonheur est le sésame de l'épreuve professionnelle de ces concours. Est-ce là un phénomène de mode comme les Instructions officielles ont le talent d'en engendrer ou une nécessité pratique qui répondrait à un besoin des élèves et dont les (futurs) enseignants se feraient l'écho, ou encore une prise de conscience des enseignants dont les élèves bénéficieraient par effet-rebond? Nous nous abstiendrons de trancher ici pour nous contenter de souligner ce qui, selon nous, est primordial dans cette démarche.

Réfléchir sur la langue c'est avant tout réfléchir sur la langue L1, prendre conscience de ses mécanismes, de sa propre façon d'exprimer tel concept, sentir que «bien » dans « il a bien trahi son frère » dépasse le cadre du simple adverbe qualitatif pour être accentué dans une intention de modalisation de l'énoncé, lequel se trouve ainsi intégralement dominé par ce jugement de l'énonciateur. Cette ouverture de l'esprit, cette sensibilité si ce n'est retrouvée du moins réveillée contribuent indiscutablement à l'avènement de cette language awareness sans laquelle tout apprentissage se résume à un travail robotisé, déshumanisé et sans âme. Les avis des auteurs sensibles à cette démarche vont tous dans le même sens (cf. annexe 3).

18 Les travaux des énonciativistes (Guillaume, Culioli, Adamczewski...) sont de précieux outils d'éveil au rôle central de l'énonciateur par lequel tout passe, qu'il s'agisse de sa localisation d'un procès dans le temps, du lien ou de la rupture de ce procès avec le moment d'énonciation, des chances de réalisation du procès ou du jugement subjectif qu'il porte sur ledit procès. Les étudiants sont les premiers surpris de leur propre prise de conscience de ces paramètres fondamentaux. Il est alors aisé de leur montrer que, par simple glissement (et non pas calque), ces phénomènes ont leur image en L2, avec d'autres outils, certes, mais bien réelle ce qui ne surprend pas outre mesure quand L1 et L2 sont issus d'une même famille linguistique.

19 Faire la chasse aux exceptions ce n'est pas tant s'évertuer à remettre en question ce qui a été établi par les prédécesseurs que vouloir évacuer l'aspect incohérent d'une démarche prescriptive qui recommande ceci sauf dans le cas de cela, sans plus de justification. La 
recherche d'invariants, de valeurs centrales ou fondamentales est une tentative de réunification de tout ce qui, en apparence, est divisé, voire opposé. Comprendre, par exemple, que le prétérit est un outil de distanciation c'est se donner les moyens de rendre compte de tous les emplois du prétérit sans se contredire ni s'exposer à devoir dresser une liste d'exceptions. Le problème est que tout n'est pas aussi facilement démontrable que la valeur centrale du prétérit et que, par excès inverse, certains linguistes n'hésitent pas à forcer la serrure pour y faire entrer leur clef quels que soient les dégâts causés par leur obstination. Il ne s'agit donc pas de fuir un abus pour se soumettre à un autre. Il faut que l'étudiant perçoive une « certaine » logique derrière les emplois aussi variés soient-ils de tel opérateur. Il est également indispensable qu'il comprenne que le linguistique n'est pas l'image codée de l'extra-linguistique et que, si la réalité a son propre mode de représentation, il en est de même pour une langue sans que celui de l'un soit le fidèle reflet de celui de l'autre. Cela dit, le linguistique est au service de l'évocation de l'extralinguistique et l'étude d'énoncés réels en contexte en est la meilleure illustration. C'est pourquoi nous avons introduit dans notre cours l'usage fréquent de cassettes vidéo enregistrées à partir de films, de reportages ou de toute autre situation de communication authentique.

20 La combinaison d'exercices d'éveil aux opérations de L1 et L2, de réflexion en commun sur tels faits de langue, d'hypothèses formulées par les étudiants et d'analyse d'énoncés authentiques a constitué l'essentiel du travail mené avec le groupe-test. Le groupetémoin, quant à lui, pratiquait apprentissage et application dans une approche descriptive des plus traditionnelles.

\section{Bilan}

L'évaluation sommative de fin d'année se compose d'un QCM en contexte (extrait d'une pièce d'A. Christie) de 40 items avec les six options présentées supra et les quatre degrés de certitude, d'un test lacunaire avec justifications à partir d'un texte complet et d'une analyse d'énoncés (ces énoncés en contexte étant le « corrigé » du QCM.)

Le seul test véritablement objectif - test de sélection - est le QCM qui nous a permis de chiffrer les résultats en prenant en compte à la fois les réponses des étudiants et le degré de certitude qu'ils attribuèrent à chacune de leurs réponses. À l'issue de nombreux calculs permettant d'obtenir le plus d'informations et la plus grande variété d'angles d'interprétation, il est ressorti deux données déterminantes quant à la comparaison entre le groupe-test et le groupe-témoin. Sur le plan quantitatif les résultats étaient similaires à savoir que, globalement, le nombre de réponses exactes était à quelques dixièmes de point près le même pour les deux groupes. Bilan a priori peu encourageant pour le travail effectué avec le groupe-test. Mais la différence s'est fait sentir très nettement au profit de ce groupe dès lors que notre observation a porté sur le plan qualitatif. En effet, les degrés de certitude du groupe-test sont très sensiblement supérieurs à ceux du groupe-témoin dans les réponses exactes. Ce qui tendrait à dire que si les réponses exactes ne sont pas plus nombreuses pour le groupe-test, elles sont cependant nettement plus sûres. (Ici encore, il serait nécessaire de détailler l'élaboration des tests et l'interprétation des résultats, ce que nous ferons lors d'une communication ultérieure.)

Le test lacunaire a connu des réponses quantitativement supérieures pour le groupe-test dans cet exercice de production semi-guidée. Mais la grande différence est plus patente encore dans les justifications accompagnant ces réponses ce qui, en soi, n'a rien de 
surprenant de la part d'étudiants préparés à comprendre de l'intérieur les mécanismes et opérations de la langue.

L'analyse d'énoncés, dont les réponses ne peuvent plus être étudiées de manière chiffrée, fait très clairement ressortir une vue plus claire et cohérente des énoncés par le groupetest et ceci, une fois de plus, s'inscrit dans la logique de leur formation au cours de l'année.

Les premières conclusions à tirer de cette expérience rejoignent finalement, et heureusement, notre hypothèse de départ à savoir que la réflexion sur L2 (et L1) est d'un apport considérablement plus précieux que la simple mémorisation mécanique de règles appliquées le plus souvent sans discernement.

De plus cette «nouvelle » activité de conceptualisation présente l'avantage pour les SPAD - et plus particulièrement nos étudiants juristes ici - de les libérer des contraintes de leur matière principale où tous leurs efforts sont tendus vers un apprentissage par cœur de textes de loi... et où il ne leur est pas demandé de la même manière de démonter les mécanismes opératoires de leur sujet d'étude, d'en élaborer leur propre système d'analyse, ni d'assurer leur autonomie d'acquisition, de pratique et d'évaluation.

\section{BIBLIOGRAPHIE}

Adamczewski, H. 1984. « Le montage d'une grammaire seconde ». Textes du Crelingua Paris :

Université de la Sorbonne Nouvelle.

Allsop, J. 1988. English Grammar. Londres : Cassell.

Bolinger, D. 1975. Aspects of Language. New York : Harcourt Brace Jovanovich.

Bonnet-Piron D. \& A.M. Kuperberg. 1990. Anglais : méthodes et techniques. Paris : Nathan.

Bouscaren, J. 1986. « Le goût du miel ». Les Langues modernes 86/2.

Byrd, P. \& B. Benson. 1992. Applied English Grammar. Boston, MA : Heinle \& Heinle.

Charlirelle. 1975. Behind the Words $\left(6^{e}\right)$. Paris : OCDL Nathan.

Close, R.A. 1964. The New English Grammar. Londres : Allen \& Unwin.

Culioli, A. 1990 Préface à Dubos, Ulrika, L'explication grammaticale du thème anglais. Paris : Nathan.

Eastwood, J. Oxford practice grammar, Oxford : O.U.P., 1992.

Eckersley, C.E. et J.M. 1960. A Comprehensive English Grammar. Londres : Longman.

Faure, G. et J. Casanova. 1971. Nouvelle grammaire anglaise. Paris : Hatier.

Gauthier, A. 1981. Opérations énonciatives et apprentissage d'une langue étrangère en milieu scolaire.

Paris : APLV.

Greenbaum, S. 1989. Good English and the Grammarian. Londres : Longman.

Groussier, M.-L. \& G. et P. Chantefort. 1994. Grammaire anglaise : thèmes construits. Paris : Hachette. 
Hawkins, E. 1984. Awareness of Language. Cambridge : Cambridge University Press.

Laruelle, Philippe. 1992. As ou like? Paris : Ellipses.

Marquet, J.-P. 1992. Mind the Steps. Paris : Ellipses.

Rivière, C. 1989. Exercices commentés de grammaire anglaise. Gap : Ophrys.

Rotgé, W. et J.-R. Lapaire. 1993. Séminaire pratique de linguistique anglaise. Toulouse : P.resses universitaires du Mirail.

Sholl, M. et M. Rollin. 1990. Guidelines to English. Paris : Magnard.

Soars, J. et L. 1986. Headway: Intermediate. Oxford : Oxford University Press.

Spankie, G.M. 1987. The Grammar you Need. Londres : Macmillan.

Spears, R. A. 1992. NTC's Dictionary of Grammar Terminology. Chicago : NTC.

Swan, M. et F. Oudart. 1983. Pratique de l'anglais de A à Z. Paris : Hatier.

Trévise, A. 1993. « Apprentissage d'une langue 2 : activités et stativités métalinguistiques». LIDIL 9, La grammaire, à quoi ça sert ? Grenoble : Université Stendhal.

\section{RÉSUMÉS}

L'apprentissage et la pratique d'une langue se sont longtemps appuyés sur l'application de règles grammaticales qui ne laissaient aucune initiative à l'apprenant. Nous avons tenté de mettre en évidence l'intérêt d'une approche explicative et énonciative qui induirait une réflexion sur les mécanismes internes à l'anglais ainsi qu'au français dans une démarche contrastive.

Language learning and language practice were originally ordered around the application of grammar rules, which deprived the learner of any possible initiative. Here we try to highlight the advantages of an explicative and utterer-centred approach conducive to active contrastive thinking about the deeper mechanisms of the English and French languages.

\section{INDEX}

Mots-clés : conceptualisation, erreur, grammaire, pratique, règle

Keywords : conceptualization, error, grammar, practice, rule

\section{AUTEUR}

\section{PHILIPPE RAPATEL}

Philippe Rapatel enseigne à la section d'anglais de la Faculté des Lettres de Besançon après avoir enseigné à la Faculté de Droit. Ses principales préoccupations sont la didactique de l'anglais et la linguistique anglaise, et plus précisément la grammaire anglaise. Ses articles (publiés dans Modèles linguistiques de Lille, le Bulletin de l'Institut de recherche en enseignement des langues et le Centre de recherche en linguistique étrangère de Besançon) sont exclusivement orientés sur ces deux axes. L'intitulé de sa thèse était : « Intérêt d'une approche explicative de type énonciatif dans l'enseignement de l'anglais à des étudiants spécialistes d'autres disciplines : appropriation et pratiques des temps, aspects et modes chez des étudiants juristes ».phil.rapatel@wanadoo.fr 